\title{
Degree of contribution (DoC) feature selection algorithm for structural brain MRI volumetric features in depression detection
}

\author{
Kuryati Kipli · Abbas Z. Kouzani
}

Received: 2 March 2014 / Accepted: 4 November 2014 / Published online: 25 November 2014

(C) CARS 2014

\begin{abstract}
Purpose Accurate detection of depression at an individual level using structural magnetic resonance imaging (sMRI) remains a challenge. Brain volumetric changes at a structural level appear to have importance in depression biomarkers studies. An automated algorithm is developed to select brain sMRI volumetric features for the detection of depression.

Methods A feature selection (FS) algorithm called degree of contribution (DoC) is developed for selection of sMRI volumetric features. This algorithm uses an ensemble approach to determine the degree of contribution in detection of major depressive disorder. The DoC is the score of feature importance used for feature ranking. The algorithm involves four stages: feature ranking, subset generation, subset evaluation, and DoC analysis. The performance of DoC is evaluated on the Duke University Multi-site Imaging Research in the Analysis of Depression sMRI dataset. The dataset consists of 115 brain sMRI scans of 88 healthy controls and 27 depressed subjects. Forty-four sMRI volumetric features are used in the evaluation.

Results The DoC score of forty-four features was determined as the accuracy threshold (Acc_Thresh) was varied. The DoC performance was compared with that of four existing FS algorithms. At all defined Acc_Threshs, DoC out-
\end{abstract}

K. Kipli $(\bowtie) \cdot$ A. Z. Kouzani

School of Engineering, Deakin University, Waurn Ponds,

VIC 3216, Australia

e-mail: kkipli@deakin.edu.au

A. Z. Kouzani

e-mail:kouzani@deakin.edu.au

K. Kipli

Department of Electronic, Faculty of Engineering, Universiti Malaysia Sarawak, 94300 Kota Samarahan,

Sarawak, Malaysia performed the four examined FS algorithms for the average classification score and the maximum classification score. Conclusion DoC has a good ability to generate reduced-size subsets of important features that could yield high classification accuracy. Based on the DoC score, the most discriminant volumetric features are those from the left-brain region.

Keywords Feature selection - Ensemble - Depression detection · Brain sMRI data $\cdot$ Volumetric features $\cdot$ Degree of contribution

\section{Introduction}

Depression is the most common mental disorder worldwide and is considered a significant health problem. Stigma and patient denial, extent of clinical experience, time limitations, and reliability of psychometrics are barriers to the clinical diagnosis of depression. Various medical diagnosis systems have been developed for different medical diagnosis problems. However, such systems are generic systems that have not been specifically designed for depression detection $[1,2]$. Thus, an automated system that is able to detect depression would assist medical experts in their decision-making process. One of the key components of a sMRI-based depression detection system is feature selection (FS) [1]. Existing FS algorithms are not tailored for the depression detection problem.

FS identifies the most useful features and reduces the feature dimensionality, while the most significant aspects of the data are preserved [3]. Feature subset selection (FSS) finds a reduced subset of features from a dataset described by the feature set [4]. Feature ranking (FR), also called feature weight- 